\title{
Developmental Stages and Clinical Conditions for Vascular Access in Pediatrics
}

\author{
Amanda Ullman and Tricia Kleidon
}

\begin{abstract}
A high-quality vascular access practice is of utmost importance in the area of pediatrics to ensure the preservation of vessel health. Pediatric clinicians must ensure that a focus on vessel health and preservation is evident from the child's earliest exposure to healthcare. The ability to ensure long-term vessel health and preservation from infancy into adulthood and the prevention of complications associated with VAD in pediatrics are key components to ensure successful, efficient, lifelong healthcare.
\end{abstract}

\section{Keywords}

Pediatric developmental stages . VHP for adolescents

\subsection{Introduction}

The vessels that children are born with are the only vessels they will have throughout their entire lifetime. Once injured, vessels rarely fully recover. Internationally, a growing number of children are reliant upon vascular access devices (VADs) for extended periods of time. Severe, life-threatening chronic health conditions, such as intestinal failure and cystic fibrosis, require children to be dependent on VADs for their entire lifespan. In addition, children undergoing treatment for complex conditions such as cancer often require additional intravenous access to treat the unintended complications of anticancer therapies. These children continue to progress through healthcare systems as adolescents and then adults, undergoing treatment for further health conditions later in life.

\subsection{Vascular Access-Related \\ 13.2 Vascular Access-Related
Anatomical, Physiological, and Developmental
Variations by Age Group and Developmental
Variations by Age Group}

\footnotetext{
Alliance for Vascular Access Teaching and Research (AVATAR) Group, Menzies Health Institute Queensland, School of Nursing and Midwifery, Griffith University, Brisbane, QLD, Australia e-mail: a.ullman@griffith.edu.au

T. Kleidon

Alliance for Vascular Access Teaching and Research (AVATAR), Queensland Children's Hospital, Brisbane, QLD, Australia

e-mail: tricia.kleidon@ health.qld.gov.au
}

A. Ullman ( $\bowtie)$
Children undergo rapid changes throughout the first 18 years of their life. The anatomical, physiological, and developmental differences between children, adolescents, and adults impact the way illnesses and diseases present. These differences determine what type of healthcare is provided at 
Fig. 13.1 Aging life cycle

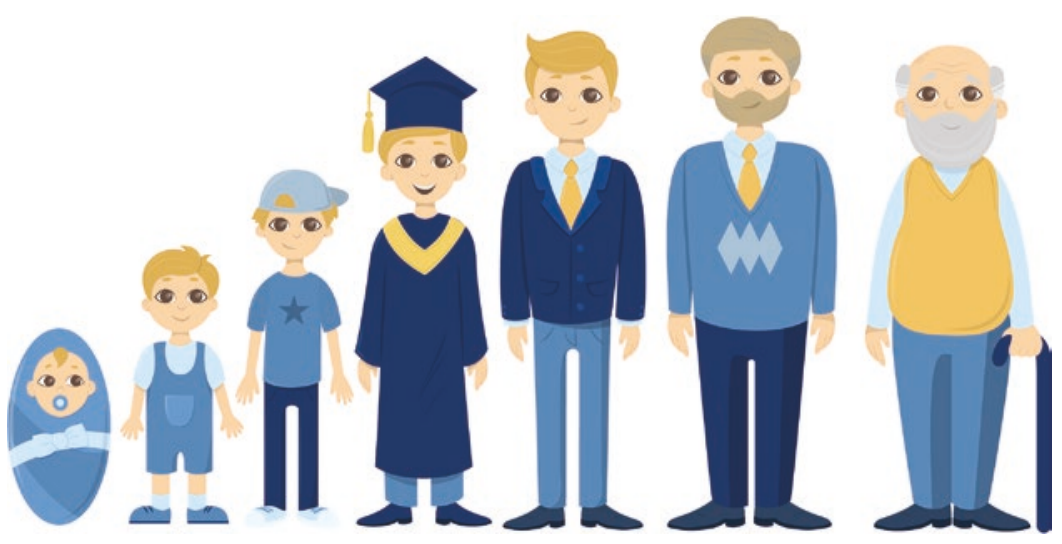

various stages for the growing child (see Fig. 13.1). These differences also have an impact on how vessel health and preservation is supported. Throughout all stages, parents and other primary caregivers should be recognized as partners with clinicians when planning, inserting, and managing VADs.

\subsubsection{Neonatal (<28 Days)}

For approximately the first week of life, the umbilical vascular network is a viable means of central venous and arterial therapy. A range of VADs are utilized for neonates in the special care or neonatal intensive care setting to facilitate therapies associated with preterm delivery, low birthweight, congenital disease, or abnormality and/or to treat infection. A definition of the common terms used in the neonatal period can be found in Table 13.1.

The neonatal vascular network continues to mature throughout the first year of life after term delivery (Mccullen and Pieper 2006). The developing vein structure includes decreased muscle diameter, meaning clinicians need to use smaller, size-appropriate catheters for both peripheral and

Table 13.1 Definitions (Rudolf et al. 2011)

\begin{tabular}{l|l}
\hline Preterm delivery & $\begin{array}{l}\text { Infant born before } 37 \\
\text { completed weeks of gestation }\end{array}$ \\
\hline $\begin{array}{l}\text { Very low birthweight } \\
\text { (VLBW) }\end{array}$ & $\begin{array}{l}\text { A baby born with a } \\
\text { birthweight of } 1500 \text { g or less }\end{array}$ \\
\hline $\begin{array}{l}\text { Extremely low } \\
\text { birthweight (ELBW) }\end{array}$ & $\begin{array}{l}\text { A baby born with a } \\
\text { birthweight of } 1000 \text { g or less }\end{array}$ \\
\hline
\end{tabular}

central devices (Franck et al. 2001). This is explored further in Chap. 14.

An important consideration for vascular access and infusion therapy is the neonates' low circulating blood volume. The total blood volume is relative to body weight and neonatal development. In the immediate post-birth neonatal period, blood volume varies from $85 \mathrm{~mL} /$ $\mathrm{kg}$ at birth rising to a peak of $105 \mathrm{~mL} / \mathrm{kg}$ by the end of the first month and then drops over the ensuing months (Sorge et al. 2016). This means the average, full-term neonate has a circulating blood volume of between 250 and $400 \mathrm{~mL}$. Careful monitoring of fluid balance in the neonatal period is critical as the infusion of multiple continuous and intermittent medications such as antibiotics, blood products, and fluid can unintentionally and dramatically increase the neonates' fluid intake, which may result in fluid overload. Conversely, excessive blood sampling can also be associated with harm, including iatrogenic anemia (Ullman et al. 2016).

Many other physiological attributes of neonates significantly increase their risk for vascular access-associated harm. An immature immune system places the neonate at significant increased risk of developing infection. Functional skin maturation continues until the second year of life; therefore, neonatal skin is thin and structurally immature (Visscher et al. 2017). A thin stratum corneum increases the risk of absorption of procedural solutions (e.g., chlorhexidine or alcohol) leading to burns and 
irritations as well as potential systemic absorption and associated harm. These solutions and other adhesive products are likely to result in adhesive-related injuries (Ponnusamy et al. 2014). Neonates are also at significant risk of other forms of skin injury associated with vascular access devices, such as pressure injuries (August et al. 2017).

\subsubsection{Infants (28 Days-1 Year)}

Infancy involves a more rapid rate of growth than at any other age (Rudolf et al. 2011). As discussed within the previous section, the infant's immature vascular network, immune system, skin structure, and circulating blood volume continue to develop over the ensuing year, providing challenges when providing vascular access and infusion therapy.

The infant's rapid growth and development also necessitates changes in pediatric vascular access practice. Rapid growth, including increased adiposity during infancy and toddler years, can make it difficult to visualize and palpate veins, making the insertion of VADs challenging. Vein visualization technology, such as ultrasound and infrared, can be useful in this age group. In particular, the insertion and management of jugular CVADs can be challenging due to increased adiposity and the limited amount of space in an infant's neck. In emergent situations when intravenous access is not possible, intraosseous (IO) infusions are sometimes necessary in this age group (Tobias and Ross 2010). The regular assessment of all VADs for signs of dysfunction and complication is especially relevant for this age group due to their inability to communicate discomfort and alert the clinician to a potential complication.

\subsubsection{Toddler (1-3 Years)}

The toddler years involve an expansion in mobility and social interaction. Within vascular access, this provides new challenges regarding procedural compliance. Various resources are available to reduce anxiety and promote compliance during VAD insertion, management, and removal procedures. Within North America, child life specialists (CLS) provide expertise in child psychosocial and cognitive development to reduce anxiety and improve experiences associated with potentially painful and stressful procedures, such as peripheral vein cannulation and CVAD management procedures (Murag et al. 2017). In other countries and facilities where CLS are not available, cognitive, distractive, behavioral, and physical strategies are used to assist pediatric patients within these situations. Localized and/or generalized pain relief during the insertion and management procedures should always be incorporated, as with other populations. The insertion of CVADs (including PICCs) into toddlers without appropriate sedation is difficult and may result in suboptimal outcomes.

Additional strategies should also be incorporated to ensure the safety of the clinician, VAD, and thereby the toddler, during mobilization and episodes of non-compliance. This includes the use of additional security devices and circumspect placement. For example, a PIVC or PICC into the leg of a newly mobile toddler is likely to be quickly dislodged.

\subsubsection{Preschool to School-Age Children (3-12 Years)}

Motor, language, and social skills continue to develop during the preschool and school years (Rudolf et al. 2011). Procedural compliance varies between children and ages. As communication improves, so does the need to ensure children are properly consulted throughout vascular access decision-making. CLS (if available) or their professional equivalent should be engaged early during the assessment and intervention phase of VAD insertion to ensure involvement. Distraction therapies are useful in this age group to assist in reducing anxiety and promoting procedural success (Murag et al. 2017). Device placement should be planned to ensure minimal disruption to the preschool and school-age child's ability to continue with usual play activities. This 
will ensure better compliance with the inserted device and reduce the risk of complications such as inadvertent dislodgement.

\subsubsection{Adolescents (13-18 Years)}

Starting from as early as 9 years of age, adolescence is characterized by a physical, psychological, and social maturity which occurs under the influence of rising hormone levels (Rudolf et al. 2011). Puberty culminates when an individual becomes physiologically capable of sexual reproduction. While many of the anatomical and physiological differences between pediatric and adult patients which impact vascular access and infusion therapy have plateaued by adolescence, there are developmental attributes which necessitate specialized care. Additionally, children with chronic illness may have exhausted many of the traditional vascular access routes by this age. This situation necessitates complex management of their vasculature, including the insertion of nontraditional vascular access routes.

As emerging adults, adolescents are generally more able to participate in decision-making about their own care and have opinions and views which can challenge those of their family and healthcare providers. It is necessary to find the most appropriate way to work with each adolescent on an individual basis, ensuring the adolescent is sufficiently involved in his/her vascular access decision-making, including choices surrounding device type, location, insertion procedure, management, and many other aspects. For adolescents with chronic health conditions, they are likely to be the expert of their own health condition, and consideration of their opinion is likely to make an important contribution to the success of the device.

\subsection{Common Pediatric Conditions Which Are Vascular Access Dependent}

The pediatric health conditions described in this section are not an exhaustive list. The list provides a short summary of common, complex, and chronic pediatric conditions that are heavily reli- ant on vascular access for management over prolonged time periods.

\subsubsection{Short Bowel (Gut) Syndrome}

Short bowel, or gut, syndrome commonly occurs in pediatric patients as a sequela of necrotizing enterocolitis, which can occur within the first few weeks of any newborn but is most commonly found in premature infants. Chronic intestinal failure results from a bowel resection that leaves a short residual length of small bowel (Nightingale and Woodward 2006). Many children with short gut syndrome are highly dependent upon vascular access for prolonged periods of time due to their reliance on parenteral nutrition. This occurs due to severe intestinal failure and/or reduced intestinal absorption so that macronutrient and/or water and electrolyte supplements are needed to maintain health/growth (Nightingale and Woodward 2006). If untreated, undernutrition and dehydration ensue, resulting in impaired growth and development.

The safe administration of parenteral nutrition commonly requires the insertion of a CVAD (M. Pittiruti et al., Pittiruti et al. 2009). International clinical practice guidelines recommend the use of parenteral nutrition if a patient absorbs less than one third of the oral energy intake, if there are high energy requirements and absorption is $30-60 \%$, or if increasing oral/ enteral nutrient intake causes a socially unacceptable amount of diarrhea or a large volume of stomal output (Nightingale and Woodward 2006).

There are many other acute and chronic causes of intestinal failure which may result in a shortor long-term dependency on parenteral nutrition (see Fig. 13.2).

There are emerging treatment strategies for end-stage intestinal failure and incurable gastrointestinal disorders, such as short bowel syndrome, including the use of intestinal transplantation (Abu-Elmagd 2006). However, consequences associated with the historically heavy immunosuppression schedules have meant that widespread use has been limited. An alternative to organ transplant in patients with short bowel syndrome is the serial transverse entero- 


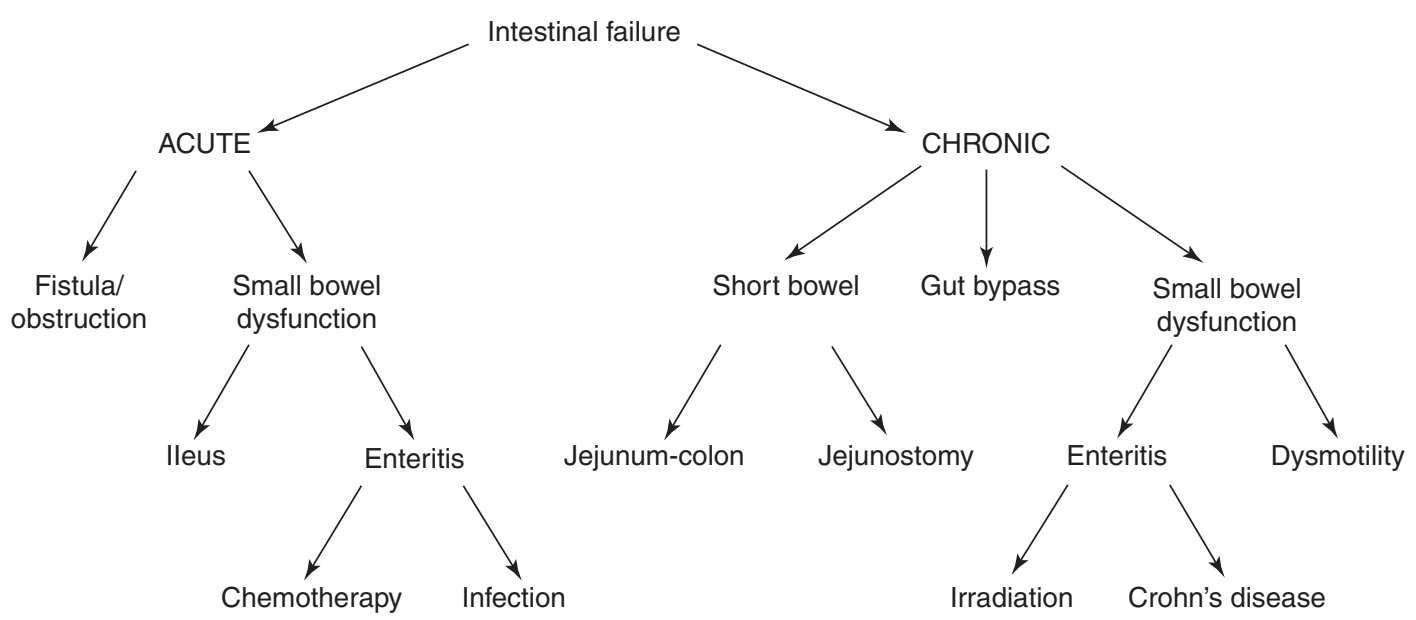

Fig. 13.2 Reasons for intestinal failure (Nightingale and Woodward 2006) (used with permission of BMJ)

plasty (STEP) procedure (Chang et al. 2006) which aims to increase intestinal absorption by optimizing intestinal function and motility, so that patients can better tolerate nutrition through the gastrointestinal tract and eventually wean from parenteral nutrition.

\subsubsection{Cystic Fibrosis}

Cystic fibrosis (CF) is a life-limiting, complex, multi-organ, congenital disorder affecting 1 in 3500 births in Australia, Europe, and the United States (Flume et al. 2010; Pedersen et al. 2015; World Health Organization 2012). CF is characterized by dehydration of the airway surface liquid and impaired mucociliary clearance (Flume et al. 2010). As a result, individuals with the disease have difficulty clearing pathogens from the lung and experience chronic pulmonary infections and inflammation.

Specialized CF care has led to a dramatic improvement in survival and quality of life (Smyth et al. 2014). An important element of early and ongoing supportive therapy is the administration of intravenous antibiotics to treat pulmonary exacerbations caused by infections. Reliable vascular access enables protocolized administration of antibiotics; a range of devices are used depending upon the patients' vessel availability, duration of treatment, severity of illness, and personal preference.

\subsubsection{Hematological Disorders}

Hematological conditions are disorders of any aspect of the blood, bone marrow, or lymph nodes. These include malignant and nonmalignant conditions which may be either acute or chronic. Chronic or life-threatening conditions frequently require repetitive vascular access due to frequent testing and the administration of treatments and supportive therapies (e.g., blood transfusions).

\subsubsection{Nonmalignant Hematological Disorders}

Common nonmalignant (benign) conditions requiring vascular access in pediatrics include:

- Sickle cell anemia: Sickle cell anemia is the most severe form of sickle cell disease, a genetic disorder of the red blood cell. Sickle cell disease is caused by abnormal hemoglobin, called hemoglobin S (or sickle hemoglobin, HbS). Several sickle cell disease genotypes exist. The most prevalent genotype, HbSS, and the much less common $\mathrm{HbS} \beta 0$-thalassemia are both commonly referred to as sickle cell anemia (SCA) because they are phenotypically very similar and are associated with the most severe clinical manifestations (Yawn et al. 2014). A wide variety of acute complications 
occur in sickle cell disease reflecting the complex pathophysiology of vaso-occlusion, infection, anemia, and infarction (Ware et al. 2017). The main treatments for the disease are red blood cell transfusions and hydroxycarbamide, with cure possible with stem cell transplantation (Ware et al. 2017).

- Aplastic anemia: Aplastic anemia (AA) is a rare and heterogenous disorder of the bone marrow in which hematopoietic stem cells get destroyed by either drugs, idiopathic or inherited autoimmune process (Barone et al. 2015). It is defined as a pancytopenia with a hypocellular bone marrow in the absence of an abnormal infiltrate, major dysplasia, or marrow fibrosis (Bhatnagar and Samarasinghe 2015). The management of AA includes platelet and red blood cell transfusions and the prevention and treatment of infections (including antibiotic prophylaxis, with severe neutropenia). Treatment choices include stem cell transplantation and immunosuppressive therapy (Bhatnagar and Samarasinghe 2015).

- Hemophilia: Hemophilia is a congenital bleeding disorder caused by a deficiency of coagulation factor VIII (in hemophilia A) or factor IX (in hemophilia B) (Srivastava et al. 2013). The deficiency is the result of mutations of the respective clotting factor gene. The severity of bleeding in hemophilia is generally correlated with the clotting factor level (Srivastava et al. 2013). The treatment of hemophilia is complex, and overall indwelling VADs are to be avoided whenever possible. However, some children with severe disease are heavily reliant on their VAD for the administration of blood components and clotting factor concentrates.

\subsubsection{Malignant Hematological Disorders}

Blood cancers, or hematological malignancies, include leukemias, lymphomas, and myeloma. The mortality rates associated with hematological malignancies have decreased over the last 30 years (Inaba et al. 2013); however, intensive, complex, vascular-access-dependent treatments are necessary. Hematological malignancies common to pediatrics include:

- Acute lymphoblastic leukemia (ALL): ALL is characterized by an overproduction of lymphocytes (immature white blood cells). These cells crowd the bone marrow, which prevent it from producing normal blood cells. Approximately $60 \%$ of patients with ALL are less than 20 years of age (Inaba et al. 2013). Treatments for ALL are highly protocolized, typically span $2-2.5$ years, and are comprised of three phases: induction of remission, intensification (or consolidation), and continuation (or maintenance). Reliable vascular access is key during all phases of treatment. Risk factors related to device complication vary dependent on the treatment phase and should be considered with device choice. In addition to treatment, supportive care is also intensive, including infection prevention and frequent blood product administration. Stem cell transplantation is an option for children with very high risk or persistent disease. Central nervous system (CNS) disease control, through a combination of systemic chemotherapy and risk-based early intensive intrathecal chemotherapy, plays a substantial role in prevention of relapse (Inaba et al. 2013).

- Acute myeloid leukemia (AML): AML involves the expansion of undifferentiated, immature, myeloid cells (Tarlock and Meshinchi 2015). Like in ALL, these cells reduce/prevent the production of normal blood cells. AML is more common in adult populations; however, pediatric AML has a genomic and epigenetic profile distinct from adult AML (Tarlock and Meshinchi 2015). Protocolized treatments and supportive care are somewhat similar in structure to ALL and are also highly vascular access dependent.

- Hodgkin's lymphoma (HL): HL is cancer of the lymphatic system, where developing lymphocytes become lymphoma cells, which include a Reed-Sternberg cell, and multiply aggressively. It is the most common of the pediatric lymphatic neoplasias, however is 
considered one of the most curable cancers, with long-term survival rates now exceeding 90\% after treatment (Mauz-Körholz et al. 2015). The staging classification of HL (I-IV) delineates severity of spread between lymph node regions and extralymphatic organs or sites (Lister et al. 1989). The treatment of pediatric HL is primarily through tailored radiation and chemotherapy (Mauz-Körholz et al. 2015).

- Non-Hodgkin lymphomas (NHL): NHL also affect the lymphatic system and originate in lymphocytes. The cancer starts in the lymphoid tissue but can originate outside the lymph nodes. Pediatric NHL are a diverse group of diseases in morphological and clinical characteristics (Sorge et al. 2016). The two main types of NHL are B-cell (in the lymph nodes in the neck, head, throat, and abdomen) and $\mathrm{T}$-cell (in the lymph nodes in the chest). Treatment of NHL varies between NHL types and is principally through traditional chemotherapy, radiotherapy, and biological therapies (e.g., monoclonal antibodies) (Sorge et al. 2016).

\subsubsection{Oncological Conditions}

While hematological malignancies such as leukemia and lymphoma are the most common individual types of cancer, over $50 \%$ of cancers originate outside of the hematological system. These include:

- Brain and CNS tumors: including astrocytoma, brain stem glioma, craniopharyngioma, ependymoma, medulloblastoma, glioma, and others

- Neuroblastoma: cancer forming in certain types of nerve tissues, frequently in the adrenal glands, neck, chest, abdomen, or spine and common in children less than 5 years of age

- Wilms tumor: cancer of the kidney(s) that is also known as the nephroblastoma, mostly occurring before 10 years of age

- Childhood rhabdomyosarcoma: cancer developing from rhabdomyoblasts which form skeletal muscles; develop in either children or young adults

- Retinoblastoma: a genetic and congenital cancer developing from immature cells of the retina, common in young children ( $<2$ years)

- Osteosarcoma: a cancerous tumor in a bone, common to teenagers and young adults

\subsection{Conclusion}

Vascular access has a fundamental role to the successful treatment and management of these complex health conditions. While the reliance on vascular access varies, a focus on vessel health and preservation is necessary throughout the entire treatment period to ensure children with these conditions can receive treatment and support for as long as necessary, including into adulthood if necessary.

\section{Case Study}

Cassie, a 13-year-old girl, is undergoing the insertion of a tunneled, cuffed CVAD to facilitate treatment and supportive therapies associated with her relapsed acute lymphoblastic leukemia (ALL). She is in the early stages of puberty, including the development of breasts.

What considerations should be made when planning this device?

\section{Summary of Key Points}

1. A high-quality vascular access practice is of utmost importance in the area of pediatrics to ensure the preservation of vessel health.

2. The anatomical, physiological, and developmental differences between children, adolescents, and adults impact the way illnesses and diseases present.

3. Developmental differences determine what type of healthcare is provided at various stages for the growing child. 
These differences also impact how vessel health and preservation is supported.

4. Throughout all stages, parents and other primary caregivers should be recognized as partners with clinicians when planning, inserting, and managing VADs.

5. Vascular access has a fundamental role to the successful treatment and management of these complex health conditions.

\section{References}

Abu-Elmagd KM. Intestinal transplantation for short bowel syndrome and gastrointestinal failure: current consensus, rewarding outcomes, and practical guidelines. Gastroenterology. 2006;130:S132-7. https://doi. org/10.1053/j.gastro.2005.09.069.

August DL, New K, Ray RA, Kandasamy Y. Frequency, location and risk factors of neonatal skin injuries from mechanical forces of pressure, friction, shear and stripping: a systematic literature review. J Neonatal Nurs. 2017;24(4):173-80. https://doi.org/10.1016/j. jnn.2017.08.003.

Barone A, Lucarelli A, Onofrillo D, Verzegnassi F, Bonanomi S, Cesaro S, Fioredda F, Iori AP, Ladogana S, Locasciulli A, Longoni D, Lanciotti M, Macaluso A, Mandaglio R, Marra N, Martire B, Maruzzi M, Menna G, Notarangelo LD, Palazzi G, Pillon M, Ramenghi U, Russo G, Svahn J, Timeus F, Tucci F, Cugno C, Zecca M, Farruggia P, Dufour C, Saracco P. Diagnosis and management of acquired aplastic anemia in childhood. Guidelines from the Marrow Failure Study Group of the Pediatric Haemato-Oncology Italian Association (AIEOP). Blood Cell Mol Dis. 2015;55:40-7. https:// doi.org/10.1016/j.bcmd.2015.03.007.

Bhatnagar N, Samarasinghe S. Diagnosis and management of childhood aplastic anaemia. Paediatr Child Health. 2015;25:343-9. https://doi.org/10.1016/j. paed.2015.04.001.

Chang RW, Javid PJ, Oh J-T, Andreoli S, Kim HB, Fauza D, Jaksic T. Serial transverse enteroplasty enhances intestinal function in a model of short bowel syndrome. Ann Surg. 2006;243:223. https://doi.org/10.1097/01. sla.0000197704.76166.07.

Flume PA, Mogayzel PJ Jr, Robinson KA, Rosenblatt RL, Quittell L, Marshall BC. Cystic fibrosis pulmonary guidelines: pulmonary complications: hemoptysis and pneumothorax. Am J Respir Crit Care
Med. 2010;182:298-306. https://doi.org/10.1164/ rccm.201002-0157CI.

Franck L, Hummel D, Connell K, Quinn D, Montgomery J. The safety and efficacy of peripheral intravenous catheters in ill neonates. Neonatal Netw. 2001;20:338. https://doi.org/10.1891/0730-0832.20.5.33.

Inaba $\mathrm{H}$, Greaves M, Mullighan CG. Acute lymphoblastic leukaemia. Lancet. 2013;381:1943-55. https://doi. org/10.1016/S0140-6736(12)62187-4.

Lister T, Crowther D, Sutcliffe S, Glatstein E, Canellos G, Young R, Rosenberg S, Coltman C, Tubiana M. Report of a committee convened to discuss the evaluation and staging of patients with Hodgkin's disease: cotswolds meeting. J Clin Oncol. 1989;7:1630-6. https://doi. org/10.1200/JCO.1989.7.11.1630.

Mauz-Körholz C, Metzger ML, Kelly KM, Schwartz CL, Castellanos ME, Dieckmann K, Kluge R, Körholz D. Pediatric hodgkin lymphoma. J Clin Oncol. 2015;33:2975-85. https://doi.org/10.1200/ JCO.2014.59.4853.

Mccullen KL, Pieper B. A retrospective chart review of risk factors for extravasation among neonates receiving peripheral intravascular fluids. J Wound Ostomy Cont Nurs. 2006;33:133-9. https://doi. org/10.1097/00152192-200603000-00006.

Murag S, Suzukawa C, Chang TP. The effects of child life specialists on success rates of intravenous cannulation. J Pediatr Nurs. 2017;36:236-40. https://doi. org/10.1016/j.pedn.2017.03.013.

Nightingale J, Woodward JM. Guidelines for management of patients with a short bowel. Gut. 2006;55:iv1-iv12. https://doi.org/10.1136/gut.2006.091108.

Pedersen MG, Jensen-Fangel S, Olesen HV, Tambe SDP, Petersen E. Outpatient parenteral antimicrobial therapy (OPAT) in patients with cystic fibrosis. BMC Infect Dis. 2015;15:290. https://doi.org/10.1186/ s12879-015-1019-4.

Pittiruti M, Hamilton H, Biffi R, Macfie J, Pertkiewicz M. ESPEN Guidelines on parenteral nutrition: central venous catheters (Access, Care, Diagnosis and Therapy of Complications). Clin Nutr. 2009;28:36577. https://doi.org/10.1016/j.clnu.2009.03.015.

Ponnusamy V, Venkatesh V, Clarke P. Skin antisepsis in the neonate: what should we use? Curr Opin Infect Dis. 2014;27:244-50. https://doi.org/10.1097/ QCO.0000000000000064.

Rudolf M, Lee T, Levene M, editors. Paediatrics and child health. Chichester: Wiley; 2011.

Smyth AR, Bell SC, Bojcin S, Bryon M, Duff A, Flume P, Kashirskaya N, Munck A, Ratjen F, Schwarzenberg SJ, Sermet-Gaudelus I, Southern KW, Taccetti G, Ullrich G, Wolfe S. European cystic fibrosis society standards of care: best practice guidelines. J Cyst Fibros. 2014;13(Suppl 1):S23-42. https://doi.org/10.1016/j. jcf.2014.03.010.

Sorge C, Mcdaniel J, Xavier A. Targeted therapies for the treatment of pediatric non-hodgkin lymphomas: present and future. Pharmaceuticals. 2016;9:28. https:// doi.org/10.3390/ph9020028. 
Srivastava A, Brewer AK, Mauser-Bunschoten EP, Key NS, Kitchen S, Llinas A, Ludlam CA, Mahlangu JN, Mulder K, Poon MC, Street A, Treatment Guidelines Working Group the World Federation of Hemophilia. Guidelines for the management of hemophilia. Haemophilia. 2013;19:e1-e47. https://doi. org/10.1111/j.1365-2516.2012.02909.x.

Tarlock K, Meshinchi S. Pediatric acute myeloid leukemia. Pediatr Clinics North Am. 2015;62:75-93. https://doi.org/10.1016/j.pcl.2014.09.007.

Tobias JD, Ross AK. Intraosseous infusions: a review for the anesthesiologist with a focus on pediatric use. Anesth Analg. 2010;110:391-401. https://doi. org/10.1213/ANE.0b013e3181c03c7f.

Ullman AJ, Keogh S, Coyer F, Long DA, New K, Rickard CM. 'True Blood' The Critical Care Story: an audit of blood sampling practice across three adult, paediatric and neonatal intensive care settings. Aust Crit
Care. 2016;29(2):90-5. https://doi.org/10.1016/j. aucc.2015.06.002.

Visscher MO, Burkes SA, Adams DM, Hammill AM, Wickett RR. Infant skin maturation: preliminary outcomes for color and biomechanical properties. Skin Res Technol. 2017;23(4):545-51. https://doi. org/10.1111/srt.12369.

Ware RE, De Montalembert M, Tshilolo L, Abboud MR. Sickle cell disease. Lancet. 2017;390:311-23. https://doi.org/10.1016/S0140-6736(17)30193-9.

World Health Organisation. Genes and human disease [Online]. 2012. Accessed 31 Aug 2017.

Yawn BP, Buchanan GR, Afenyi-Annan AN, et al. Management of sickle cell disease: summary of the 2014 evidence-based report by expert panel members. JAMA. 2014;312:1033-48. https://doi.org/10.1001/ jama.2014.10517.

Open Access This chapter is licensed under the terms of the Creative Commons Attribution 4.0 International License (http://creativecommons.org/licenses/by/4.0/), which permits use, sharing, adaptation, distribution and reproduction in any medium or format, as long as you give appropriate credit to the original author(s) and the source, provide a link to the Creative Commons license and indicate if changes were made.

The images or other third party material in this chapter are included in the chapter's Creative Commons license, unless indicated otherwise in a credit line to the material. If material is not included in the chapter's Creative Commons license and your intended use is not permitted by statutory regulation or exceeds the permitted use, you will need to obtain permission directly from the copyright holder.

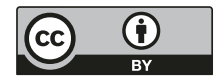

